\title{
Author Correction: Visualization of the strain-induced topological phase transition in a quasi-one-dimensional superconductor $\mathrm{TaSe}_{3}$
}

Chun Lin (D), Masayuki Ochi, Ryo Noguchi (D), Kenta Kuroda (D), Masahito Sakoda (D), Atsushi Nomura, Masakatsu Tsubota, Peng Zhang (D), Cedric Bareille, Kifu Kurokawa, Yosuke Arai, Kaishu Kawaguchi, Hiroaki Tanaka (D), Koichiro Yaji, Ayumi Harasawa, Makoto Hashimoto (D), Donghui Lu DD, Shik Shin (D), Ryotaro Arita (D), Satoshi Tanda and Takeshi Kondo (D) Correction to: Nature Materials https://doi.org/10.1038/s41563-021-01004-4, published online 20 May 2021.

In the version of this Article originally published, in the first sentence of the Methods section, the unit of lattice constants $a, b$ and $c$ was incorrect as $\AA^{-1}$; it should have been $\AA$. This has now been corrected.

Published online: 23 June 2021

https://doi.org/10.1038/s41563-021-01056-6

( ) The Author(s), under exclusive licence to Springer Nature Limited 2021 